\title{
The within-list distributed practice effect: Tests of the varied context and varied encoding hypotheses
}

\author{
ANN STASH MASKARINEC and CHARLES P. THOMPSON \\ Kansas State University, Manhattan, Kansas 66506
}

\begin{abstract}
The present studies provided separate tests of the varied context and varied encoding hypotheses of the MP-DP effect. The investigation of varied encoding used an incidental learning procedure in which the nature of the orienting task was manipulated such that the subject attended to different attributes of words (varied encoding) or only one attribute (same encoding). While the prediction that the recall of MP-DP items should be comparable under comparable levels of encoding was not supported, differences were obtained in recall of items under same and variable orienting task conditions. An MP-DP effect was obtained under the incidental learning procedure. Tests of varied context involved the presentation of target items in list contexts which were the same or different from list contexts on previous occurrences of the item. The prediction that recall of items surrounded by different context should exceed that of items surrounded by the same context was not supported.
\end{abstract}

It has been clearly and repeatedly established that distributed repetition (DP) of verbal events within $a_{1}$ single trial produces better recall performance than does massed (MP) repetition (e.g., Underwood, 1970). Although a mass of evidence has accumulated (see reviews by Hintzman, 1974; Melton, 1970), no convincing theoretical explanation of the phenomenon has emerged. The most widely investigated proposal has been the context hypothesis put forth by Melton $(1967,1970)$. The major points of this paper will be to emphasize that Melton's hypothesis contains two separable theoretical alternatives and to make some initial tests of those alternatives. Further we will introduce a procedure which should sharply reduce the effects of one alternative.

The first alternative emphasizes the role of list context effects. Melton (1970) suggested that distributed repetition permits a greater number of different cues from surrounding items to be associated with the repeated item than does massed repetition and that these additional cues aid retrieval. The second alternative emphasizes the assumption that there are many different ways a to-be-remembered (TBR) word can be encoded in memory (Melton, 1967). It follows that the greater the number of different encodings given for the word during the study phase, the greater the number of different ways in which it might be retrieved (Hintzman,

This research was based upon the first author's doctoral dissertation, completed at Kansas State University, Manhattan, Kansas, and was supported by a predoctoral fellowship in experimental psychology from National Institute of Mental Health Experimental Training Grant MH-08359-06. The first author's present mailing address: Kansas Center for Mental Retardation and Human Development, University of Kansas Medical Center, Building 37, Room 16, Kansas City, Kansas 66103.
1974). Given these assumptions, the variable encoding hypothesis predicts that the MP-DP effect is the result of the increased variability in the encoding of a word under distributed conditions.

We will distinguish between these two theoretical alternatives by naming them the "varied context" and the "varied encoding" hypotheses, respectively. It should be reemphasized that in the varied context hypothesis the focus of attention is primarily on the associations formed between list items, while in the varied encoding hypothesis the unit of analysis is the individual word. The varied encoding hypothesis predicts the MP-DP effect even in the absence of the subject's attempt to organize words into subjective units, that is, to use list context. However, most current investigators of varied encoding in MP-DP have addressed themselves to the combined effects of change of context upon the encoding of the TBR information (e.g., Gartman \& Johnson, 1972; Madigan, 1969; Glenberg, Note 1). Since it is clear that one hypothesis emphasizes word association while the other stresses individual word encoding, it seems appropriate that separate tests be performed investigating the effects of context and encoding. Experiment 1 was designed to test the effects of list context, whereas Experiment 2 attempted to eliminate any contribution of context while simultaneously assessing the effect of varied encoding. As will be seen later, the second experiment also provides relevant evidence for yet another proposed explanation of the MP-DP effect.

\section{EXPERIMENT 1: A TEST OF THE VARIED CONTEXT HYPOTHESIS}

The varied context hypothesis states that the importance of varying the context is to increase the number of 
cues for retrieving an item; these cues are usually supplied by association to immediately surrounding list items (Tulving, 1968). The varied context hypothesis was tested by controlling the list context surrounding a specific target word. Target words were presented three times in a list. On each occurrence a target item was presented in a context of three words (defined as the two preceding and one following words) which was either the same or different from the list context on its previous occurrence. In one condition, a target item was surrounded by the same context on each occurrence $\left(\mathrm{SC}_{3}\right)$. In another condition, a different context surrounded the target item on each occurrence and each list context appeared only once in the list $\left(\mathrm{DC}_{1}\right)$.

The varied context hypothesis predicts that recall for the target items surrounded by different context should exceed the recall of target items surrounded by the same context. However, it is clear that in the same context condition $\left(\mathrm{SC}_{3}\right)$ the subject is exposed to the context words three times in the list, while in the different context condition $\left(D_{1}\right)$ the context words are presented only once. Repeating context items may facilitate forming stronger associations. Thus, the $\mathrm{SC}_{3}$ and the $\mathrm{DC}_{1}$ comparison may not be appropriate. $\mathrm{A}$ third condition was necessary to control for strength of list context effects. In this condition the different context words were presented three times in the list $\left(\mathrm{DC}_{3}\right)$. A fourth condition was also included where all repetitions of the target item were presented consecutively (MP). This condition provides a measure against which one can compare the facilitatory effects of distributed practice. Several (i.e., six) MP items were incorporated in the list but only one of these items was arbitrarily designated as the target item. The MP target item was surrounded by different (once-presented) list items.

While the critical comparison for this hypothesis is between the $\mathrm{SC}_{3}$ and $\mathrm{DC}_{3}$ conditions, which reveal the effect of context, a comparison between the $\mathrm{DC}_{3}$ and $\mathrm{DC}_{1}$ conditions should reveal the effect of strengthening of list context effects. In both comparisons the recall for target items should be best in the $\mathrm{DC}_{3}$ condition because the target items receive benefit from triple presentation, as well as the greatest opportunity to form a large number of associations with other list items.

\section{Method}

Subjects. Fifty-four general psychology students, who volunteered their services for extra credit, participated in this experiment.

Experimental conditions. There were four conditions in the experiment, with target items in each condition appearing three times in each list. All conditions were described in the previous section. Briefly, the target items in the MP condition were presented in adjacent positions in the list. In the $\mathrm{SC}_{3}, \mathrm{DC}_{1}$, and $\mathrm{DC}_{3}$ conditions, the target items were distributed in the list and were presented in a context of three words which was either the same or different from the list context on its previous occurrences.

Materials. Twenty-four lists were constructed from the Toronto word pool (Murdock, 1968). Each list contained all four conditions and used 40 different words. Each list was composed of 28 experimental items ( 4 target items and 24 context items) and 12 filler items. Each target item appeared three times in a list. Context items in the MP and $\mathrm{DC}_{1}$ conditions appeared three times in the list. Five filler items were used to control for primacy effects and 8 for recency effects. Of the 12 filler items, 3 were used as MP items in the body of the list, 1 was used as an MP item in primacy positions, 1 was used as an MP item in recency positions, and the remaining 7 were once-presented items in primacy and recency positions. There was a minimum lag of 10 items between repetitions of experimental items. To accommodate experimental and filler items, a total of 82 list positions was necessary.

Experimental design. A within-subjects design was used, with each subject receiving four lists of words, and each list containing all four conditions. The ordering of conditions was randomized so that each condition appeared equally often in the first and second half of the list. Six sets of four lists were reconstructed so that new words were used for target items in each condition. Therefore, across the six sets of four lists, 24 different words were sampled as target items for any one condition.

Procedure. The subjects were tested in groups of four or five. The lists of words were presented by tape recorder at a $3-\mathrm{sec}$ rate. At the end of each list, the subjects were asked to free recall, writing the words in a prepared answer booklet. Three minutes were allowed for the recall of each list.

\section{Results}

The data of primary interest were the mean probability of correct recall of target words by each subject in each condition in each list. Table 1 shows the mean probability of target words in each condition. A withinsubjects analysis of variance was performed on the data. The analysis revealed a significant main effect of experimental conditions $[\mathrm{F}(3,159)=26.63, \mathrm{MSe}=.94]$. A $\mathrm{p}<.05$ criterion was used for all statistical tests performed in this report. A subsequent Newman-Keuls test indicated that the recall of MP target items was reliably different from the recall in the three DP conditions. However, the DP conditions were not reliably different from each other. An analysis of variance performed on the recall of MP target items appearing in the first and second half of the list showed no reliable serial position effects.

An analysis of variance was also performed on the probability of recall of the once- or thrice-presented context words. The total number of context words recalled by each subject in each condition was converted to a probability-of-recall score. The probability score was obtained by dividing the total number of different context words in each condition. For example, if a

Table 1

Mean Probability of Recall of Words in Each Condition

\begin{tabular}{lcccc}
\hline & \multicolumn{4}{c}{ Experimental Conditions } \\
\cline { 2 - 5 } & $\mathrm{MP*}$ & $\mathrm{SC}_{3}{ }^{* *}$ & $\mathrm{DC}_{\mathbf{1}} \dagger$ & $\mathrm{DC}_{3}{ }^{\dagger} \dagger$ \\
\hline Target Words & .24 & .53 & .60 & .60 \\
Context Words & .21 & .51 & .20 & .48 \\
\hline
\end{tabular}

${ }^{*} M P=$ massed practice

**SC$C_{3}=$ same context presented three times

$T D C_{1}=$ different context presented once

$+{ }^{\prime} D C_{3}=$ different context presented three times 
subject recalled two context words surrounding the $\mathrm{SC}_{3}$ target item, his probability score would be $2 / 3$ or 66 . Table 1 also shows the mean probability of recall of context words in each condition. The analysis performed on these data showed that the recall of the thricepresented context items was superior to that of the once-presented items $[F(3,159)=99.83, \mathrm{MSe}=151.14]$. A Newman-Keuls test indicated that the MP and the $\mathrm{DC}_{1}$ conditions were not reliably different from each other, but both were reliably different from the $\mathrm{SC}_{3}$ and $\mathrm{DC}_{3}$ conditions. The recall in the latter two conditions did not differ significantly from each other. The calculation of power in this experiment revealed a $\theta^{\prime}$ coefficient $\left[(a=.05)=4.31, v_{1}=3, v_{2}=54\right]$, corresponding to a power of 99 .

\section{Discussion}

The context hypothesis predicts that the recall for words surrounded by different context $\left(\mathrm{DC}_{3}\right)$ should be greater than for words surrounded by the same context $\left(\mathrm{SC}_{3}\right)$. With such a striking difference in list environment, the context hypothesis must predict differences in the probability of recall of the target words. Yet no significant differences were found between these critical conditions in this experiment. The failure to obtain differences cannot be due to the possibility that the present study did not provide a powerful test, since the power analysis clearly presents evidence to the contrary.

The argument might be made that controlling immediately adjacent positions is not sufficient to eliminate context effects. While it is true that context effects may not be eliminated by this manipulation, the magnitude of the MP-DP effect should be sharply reduced. However, there was no reliable reduction of the MP-DP effect. Thus, the results of this experiment suggest that the contribution of list context to the MP-DP effect is minimal.

The varied context hypothesis was investigated further in a second experiment. An attempt was made to eliminate context effects while simultaneously testing the varied encoding hypothesis. Specifically, it was assumed that associations between adjacent items would be essentially eliminated in the incidental learning task (used to manipulate encoding) in the next experiment. A simple demonstration of the MP-DP effect in the second experiment would suggest that something other than context must be producing the phenomenon.

\section{EXPERIMENT 2: A TEST OF THE VARIED ENCODING HYPOTHESIS AND AN ALTERNATE (ATTENTION) HYPOTHESIS}

The major purpose of this experiment was to provide a test of the varied encoding hypothesis. However, latency data gathered in this experiment also provided relevant information for an alternate (attention) hypo- thesis about the MP-DP effect. The research relevant to each hypothesis is presented separately below.

\section{The Varied Encoding Hypothesis}

As noted previously, the assumption of the varied encoding hypothesis is that spaced presentation of a verbal item facilitates recall because spacing provides the opportunity for different coded responses of an item to be elicited on different occasions. To date, nearly all studies purporting to test the varied encoding hypothesis have employed a procedure which involves the repetition of homographs (e.g., Gartman \& Johnson, 1972; Johnston, Coots, \& Flickinger, 1972; Madigan, 1969; Nelson, 1971). Context is manipulated so that the meaning of the homograph either remains the same or is changed. We contend that changing the meaning of the homograph is an inappropriate test of the varied encoding hypothesis, since the recall of either or both of two different words (the two meanings of the homograph) must be better than recall of one of those words.

In the present experiment, we decided to manipulate the encoding of words by varying the nature of an orienting task in an incidental learning paradigm. The orienting task provides reasonable control over the manner in which a word is encoded. The incidental learning task greatly reduces, and possibly eliminates, context effects (e.g., interitem associations) and idiosyncratic methods of coding words for later recall. Specifically, in this experiment, the subjects responded to statements about target words which stressed physical, syntactic, or semantic attributes. Further, over three presentations of the critical words, the nature of the orienting task was manipulated so that the subject was required to attend to all three different attributes of MP and DP items (which is assumed to result in the varied encoding of the word) or the subject was required to attend to only one attribute of the MP and DP items (which is assumed to result in encoding along the same attribute). This experimental manipulation should provide tests of two predictions of the varied encoding hypothesis: (1) If both MP and DP items receive comparable variation in encoding (i.e., MP and DP items coded along three different attributes or MP and DP items coded along one attribute), recall for MP and DP items should be comparable and the MP-DP effect should be eliminated. (2) The recall for items variably encoded (i.e., the subject's attending to three different attributes of a word) should exceed the recall of items which do not receive the benefit of this variation (subject's attending to one attribute of a word).

\section{The Attention Hypothesis}

The evidence relevant to the attention hypothesis was obtained purely as a result of a methodological precaution. The subjects' reaction times to each sentence were obtained so that any confounding effects due to differential processing time between MP and DP items could be assessed. Clearly, these data could also be used 
to evaluate the implications of the attention hypothesis for the MP.DP effect.

In brief, the attention hypothesis was formulated by Shaughnessy, Zimmerman, and Underwood (1972), who proposed that MP-DP differences can be attributed to the decrease in the total time that the subject attends to MP as compared to DP items. Note that while the first study involved a list learning situation, the subjects in Experiment 2 were asked to attend to a statement about a specified target item and to make the appropriate response. Assuming that the time taken by a subject to respond to a sentence represents processing/attention time, these authors would predict that if an MP-DP effect is obtained in recall performance, this difference should be accompanied by longer reaction times to sentences under DP conditions.

\section{Method}

Subjects. One-hundred and twenty general psychology students, who volunteered their services for extra credit, participated in this experiment.

Materials. The target items in each sentence were taken from the word pool described in Experiment 1. A mixed-list design was used such that each list contained sentences with target items representing all four experimental conditions. In each list, sentences were constructed which used 12 different words as target items and 12 as filler items. The target items occurred three times in a list. Four sentences using filler items were used to absorb primacy effects and eight for recency effects. Of these filler items, 4 occurred twice in the list and 4 occurred once. Placement of the twice-presented filler items was random. Where necessary, sentences with filler items were inserted in the list to produce a minimal lag of 10 . To accommodate sentences with target and filler items, 52 list positions were necessary.

Orienting tasks. This experiment used three different orienting tasks: (1) a rhyme task (physical feature), (2) a syntax task (syntactical feature), and (3) a meaning task (semantic feature). In the rhyme task the subject was asked to judge (yes/no) whether a certain target word rhymed with another word; in the syntax task he was asked to judge whether a target word was a verb, adjective, or noun; in the meaning task he was asked to judge if a target word was a synonym of another word. Under the varied encoding conditions a target word (e.g, teacher) was presented three times in a list. On the first presentation, for example, the subject was asked to judge if "teacher is a verb." On the second occurrence he was asked to judge if "teacher thymes with preacher," and on the third he was asked to judge if "teacher means instructor." Since the subject must focus on three different attributes of the word to meet the demands of the task, it was assumed that he had coded the word along those three attributes, resulting in the "varied encoding" of that word. Under the same encoding conditions, the subject was asked to focus only on one attribute, for example, the rhyme feature. On the first presentation of the word the subject was asked to judge if "teacher rhymes with preacher," on the second presentation if "teacher rhymes with garden," and on the third presentation if "teacher rhymes with creature." Since the subject must focus only on one attribute of the word to meet the demands of the task, it was assumed that he had coded the word along only the same attribute, resulting in the "same" encoding of that word.

Experimental conditions. There were four experimental conditions represented by the factorial combination of type of presentation (massed, distributed) and the variation in orienting tasks (same encoding, one type of task; varied encoding, three types of tasks). In the DP conditions the sentences containing the target items were distributed through the list with a minimum lag of 10 .

Experimental design. Each subject received only one list. Twenty-four lists were constructed so that an adequate sample of target words (72) was used in each of the four conditions. The ordering of conditions in each list was randomized so that sentences containing target items from each condition appeared equally often in both halves of the list. To eliminate the possibility of response bias, responses were counterbalanced over subjects and over lists so that half the judgments in each of the four conditions had "no" as the correct response and half had "yes" as the correct response. The location of the (yes/no) response was also counterbalanced over subjects and lists so that there were an equal number of yes and no responses in the first, second, and third sentences for target items in the experimental conditions. Furthermore, in the varied encoding conditions the rhyme, syntax, and meaning sentences appeared equally as often as the first, second, and third sentence.

Procedure. Each subject was given a pretest consisting of 10 sentences similar in form to the experimental sentences. No sentence contained any word which was used in the experimental sentences. The pretest was given to remind the subjects of the definitions of noun, verb, adjective, rhyme, and synonym. At this time the subjects were told that the first word they heard was the target word; this was the word we were interested in and he was to keep this word in mind when making his judgment.

Each subject was tested individually. The sentences (e.g., teacher rhymes with preacher) were presented by tape recorder. The presentation time for sentences ranged between 1.5 and $2.0 \mathrm{sec}$. The sentences were manually presented to the subject approximately 1.5 to $2.0 \mathrm{sec}$ after he made a response to the previous sentence. The entire experimental session, which included sentence presentation and the subject's response to each sentence, was recorded. The subject was told that we were interested in how long it took him to make the correct response. At the end of the list the subject was given a surprise memory test. He was asked to recall those items which were identified as target items. Three minutes were allowed for written free recall. Subjects were asked not to inform other classmates about the surprise memory test. Since the subjects were drawn from four psychology classes containing approximately 150 members, intercommunication seemed to pose no major problem. In addition, the experiment was conducted over a period of two semesters.

\section{Results}

The varied encoding hypothesis. The data of primary interest for the varied encoding hypothesis were the mean probability of correct recall of target words by each subject in each experimental condition. Table 2 presents the mean probability of correct recall in each condition. A within-subjects analysis of variance was performed on these data. The analysis revealed a significant main effect of type of practice $[F(1,119)=55.17$, $\mathrm{MSe}=.59]$. That is, the recall of items under MP $(M=.24)$ was found to be inferior to the recall of items under DP $(M=.41)$. A significant main effect of type of orienting task was also obtained $[F(1,119)=5.66$,

Table 2

Mean Probability of Recall of Words in Each Condition

\begin{tabular}{ccc}
\hline & \multicolumn{2}{c}{ Type of Practice } \\
\cline { 2 - 3 } Orienting Task & Massed & Distributed \\
\hline Same & .21 & .38 \\
Variable & .27 & .44 \\
\hline
\end{tabular}


MSe $=.68]$. This effect reflects the better recall performance for items under the varied orienting task conditions $(M=.36)$ as compared with items under the same orienting task conditions $(M=.29)$. No significant interaction was obtained between type of practice and orienting task $(F<1)$.

The attention hypothesis. The pertinent data for the attention hypothesis were the reaction time measures. Reaction time (RT) was defined as the time elapsing between the offset of the sentence (last-heard phoneme of the last word in the sentence) and the onset of the response (the first-heard phoneme of the words yes or no). These RTs were subjected to a 2 (MP-DP) by 2 (orienting task-same, varied) by 3 (sentence typerhyme, syntax, meaning) by 2 (response-yes, no) within-subjects analysis of variance. Since there were no errors, the RTs for all experimental sentences were used in the analysis. However, due to technical difficulties, the data of only 109 of the 120 subjects were used.

Contrary to the prediction of the attention hypothesis, the analysis did not reveal a reliable main effect of type of practice $[\mathrm{F}<1, \mathrm{MSe}=8.21]$, nor did any interaction involving type of practice approach significance. In other words, attention time was not reliably longer for sentences under DP conditions. However, significant differences were found between the same and varied encoding conditions $[F(1,108)=10.53$, $\mathrm{MSe}=8.32]$. The mean RT was $.63 \mathrm{sec}$ for sentences under the same encoding conditions and $.67 \mathrm{sec}$ for sentences under the varied encoding conditions.

\section{Discussion}

The varied encoding hypothesis. The important finding obtained in this study was that the MP-DP effect occurred with an incidental learning task. As noted in the discussion of Experiment 1, a simple demonstration of the MP-DP effect in incidental learning suggests that something other than list context must be producing the phenomenon. Thus, the present data, when combined with those obtained in Experiment 1, suggest that list context may not be an important factor in the MP-DP effect.

The other important findings concern the role of varied encoding. The prediction of the varied encoding hypothesis concerning the MP.DP effect was that recall performance under MP and DP conditions should be comparable under comparable variation in orienting tasks. That is, no MP.DP effect should be obtained. Clearly, the data in this experiment do not support this prediction, since an overwhelming MP-DP effect was obtained. Also, the RT measures show that the superior performance for target words in sentences under DP conditions cannot be attributed to increased time spent on these sentences. No significant differences were found between sentences under MP and DP conditions. Therefore, it is obvious that these results do not provide support for a critical prediction of the hypothesis.

A second prediction of the varied encoding hypothesis was that recall for items under the varied orienting task condition should be superior to the recall of items under the same orienting task condition. While this prediction was borne out by the recall data, the RT analysis revealed a finding which must be considered before the recall data is interpreted as providing support for the above prediction. The analysis showed that the RTs were reliably longer for sentences under the variable orienting task condition than for sentences under the same orienting task condition. Thus, the superior recall performance for words in the former condition may be due, at least in part, to longer processing time for these sentences. Therefore, it is not clear from these data that variation in encoding resulting from variation in orienting task is solely responsible for the better recall of the words.

Thus, the results of this experiment suggest that varied encoding, as defined by variation in orienting tasks, may not play a major role in the MP-DP effect. In addition, the data in the experiment do not unambiguously demonstrate that the superior performance under the varied orienting task condition results exclusively from variation in encoding. Taken together, the results may lead one to question whether experimenter-induced variation in encoding had any effect upon recall performance in this experiment.

The attention hypothesis. In general terms, Shaughnessy, Zimmerman, and Underwood (1972) predicted that recall should be better for any item which has the benefit of increased attention. More specifically, they would predict that if an MP-DP effect is obtained in recall performance, this difference should be accompanied by longer RTs to sentences under the DP condition. In the present experiment, the analysis of recall performance did reveal an MP-DP effect. However, the RT analysis failed to find any significant difference in RT between MP and DP sentences, nor did any interaction involving type of practice approach significance. Clearly, these data do not support the prediction and one may assert that the attention hypothesis does not seem to be a viable explanation of the MP-DP effect in this experimental situation.

\section{GENERAL DISCUSSION}

The studies reported here contribute to our knowledge of the MP-DP effect in three respects. First, we made a distinction between two theoretically separable components of the general context hypothesis and made some initial tests of those alternatives. The data from our experiments do not tend to support either the varied context or the varied encoding hypotheses.

Second, we found a strong MP-DP effect under an incidental learning procedure. The demonstration of the 
effect under conditions in which associations between adjacent items are essentially eliminated suggests that something other than context (as defined here) must be producing the effect. Taken together, these data suggest that the contribution of the context effect to the MP-DP phenomenon may be negligible.

Third, our experiment provided data concerning the attention hypothesis. While the data were obtained as the result of a methodological precaution, they are pertinent to the hypothesis and do not lend any support for it as an explanation of the MP-DP effect.

In summary, the demonstration of an MP.DP effect in an incidental leaming paradigm, together with the rest of the data from these experiments, suggests that investigators begin looking in other directions for the explanation of this phenomenon. The explanation for the MP-DP effect seems more likely to be found in something similar to a simple spacing hypothesis (Hintzman, 1974) or an organizational hypothesis (Jacoby, Note 2).

\section{REFERENCE NOTES}

1. Glenberg, A. M. Retrieval factors and the lag effect. Paper presented at the Mathematical Psychology Meeting, Ann Arbor, August 1974.

2. Jacoby, L. L. Effects of spacing repetitions: The role of trace interaction. Paper presented at the meeting of the Psychonomic Society, Denver, November 1975.

\section{REFERENCES}

Gartman, L. M., \& Johnson, N. F. Massed versus distributed repetition of homographs: A test of the differential encoding hypothesis. Journal of Verbal Leaming and Verbal Behavior, 1972, 11, 801-808.

Hintzman, D. L. Theoretical implications of the spacing effect. In R. L. Solso (Ed.), Theories in cognitive psychology: The Loyola symposium. Potomac, Md: Lawrence Erlbaum, 1974.

Johnston, W. A., Coots, J. H., \& Flickinger, R. C. Controlled semantic encoding and the effect of repetition lag on free recall. Journal of Verbal Learning and Verbal Behavior, 1972, 11. 784-788.

Madigan, S. W. Intraserial repetition and coding processes in free recall. Journal of Verbal Learning and Verbal Behavior, 1969, 8, 828-835.

Meltow, A. W. Repetition and retrieval from memory. Science, 1967, 158, 532.

Melton, A. W. The situation with respect to the spacing of repetitions and memory. Journal of Verbal Learning and Verbal Behavior, 1970, 9, 596-606.

Murdock, B. B. Modality effects in short-term memory: Storage or retrieval? Joumal of Experimental Psychology, $1968,77,79-86$.

Nelson, L. Effects of subjective organization on single trial free recall of homographs under massed versus distributed practice. Dissertation Abstracts International, 1971, 33(3-B), 1310.

Shaughnessy, J. J., Zimmerman, J., \& Underwood, B. J. Further evidence on the MP-DP effect in free recall learning. Journal of Verbal Learning and Verbal Behavior, 1972, 11, $1-12$.

Turving, E. Theoretical issues in free recall. In T. R. Dixon \& D. L. Horton (Eds.), Verbal Behavior and General Behavior Theory. Engelwood Cliffs, N.J: Prentice-Hall, 1968. Pp. 2-36.

UNDERWOOD, B. J. A breakdown of the total-time law in free recall learning. Joumal of Verbal Learning and Verbal Behavior, 1970, 9, 573-580.

(Received for publication February 4, 1976; revisions accepted April 29, 1976.) 